\title{
THE STRUCTURE OF SOLUTIONS OF A SEMILINEAR ELLIPTIC EQUATION
}

\author{
KUO-SHUNG CHENG AND TAI-CHIA LIN
}

\begin{abstract}
We give a complete classification of solutions of the elliptic equation $\Delta u+K(x) e^{2 u}=0$ in $\mathbb{R}^{n}, n \geq 3$, for some interesting cases of $K$.
\end{abstract}

\section{INTRODUCTION}

In this paper we shall investigate the structure of the set of all entire solutions of the equation

$$
\Delta u+K e^{2 u}=0
$$

in $\mathbb{R}^{n}, n \geq 3$, where $\Delta=\sum_{i=1}^{n} \partial^{2} / \partial x_{i}^{2}$ and $K \neq 0$ is a given locally Hölder continuous function on $\mathbb{R}^{n}$. We shall consider mainly two types of solutions: $u_{c}(x)$ denotes a solution of (1.1) satisfying

$$
u_{c}(x) \rightarrow c \quad \text { as }|x| \rightarrow \infty
$$

and $U(x)$ denotes the "maximal solution"

$$
U(x)=\sup \{u(x) \mid u \text { is a solution of }(1.1)\} .
$$

In this paper we shall consider mainly three questions:

(a) When do solutions $u_{c}$ and $U$ exist?

(b) What are the relations between $K$ and $U$ ?

(c) When do we have $u=u_{c}$ or $u=U$ for every solution $u$ of (1.1)?

Equations (1.1) arises from physics and geometry. When $n=3$ and $K(x)=$ $1 / 1+|x|^{2},(1.1)$ is known as Eddington's equation, which was used to model globular clusters of stars. When $n=2,(1.1)$ is known as the conformal Gaussian curvature equation on $\mathbb{R}^{2}$. Let $(M, g)$ be a Riemannian manifold of dimension 2 and $K$ be a given function on $M$. The following question has been raised: can we find a new metric $g_{1}$ on $M$ such that $K$ is a Gaussian curvature of $g_{1}$ and $g_{1}$ is conformal to $g$ (that is, $g_{1}=\varphi g$ for some positive function $\varphi$ on $M$ ) ? If we write $\varphi=e^{2 u}$, then this is equivalent to the problem of solving the elliptic equation

$$
\Delta_{g} u-k+K e^{2 u}=0
$$

Received by the editors May 31, 1989 and, in revised form, March 31, 1990. 1980 Mathematics Subject Classification (1985 Revision). Primary 35J60; Secondary $45 G 10$. Work was supported by the National Science Council of the Republic of China. 
on $M$, where $\Delta_{g}$ and $k$ are the Laplace-Betrami operator and the Gaussian curvature on $M$ in the $g$-metric respectively. In case $M$ is compact, equation (1.4) has been considered by many authors and we refer the reader to the monograph by Kazdan [K] for details and references. In case $M$ is complete and noncompact, the first natural case seems to be $M=\mathbb{R}^{2}$ and $g$ is the usual Euclidean metric. In this case, equation (1.4) reduces to equation (1.1) with $n=2$. We refer the reader to Cheng and Ni [CN1] for details and references about equation (1.1) when $n=2$.

The first nonexistence result concerning equation (1.1) seems due to Oleinik [O]. She proved

Theorem A. Suppose that $K \leq 0$ on $\mathbb{R}^{n}$ and $|K(x)| \geq C|x|^{-p}$ for $|x|$ large for some constant $p<2$ and sone constant $C>0$. Then (1.1) possesses no entire solution.

The first existence result concerning equation (1.1) seems due to $\mathrm{Ni}$ [N1]. He obtained

Theorem B. Suppose that $|K(x)| \leq C\left|x_{1}\right|^{-p}$ for $\left|x_{1}\right|$ large and uniformly in $x_{2}$ for some $p>2$, where $x=\left(x_{1}, x_{2}\right)$ and $x_{1} \in \mathbb{R}^{m}, x_{2} \in \mathbb{R}^{n-m}, m \geq 3$. Then (1.1) possesses infinitely many bounded solutions.

Later on, Kusano and Oharu [KO] slightly extended Ni's result. They obtained

Theorem C. Suppose that $|K(x)| \leq \widetilde{K}\left(\left|x_{1}\right|\right)$ on $\mathbb{R}^{n}$ and $\int_{0}^{\infty} t \widetilde{K}(t) d t<\infty$, where $x=\left(x_{1}, x_{2}\right)$ is as in Theorem B. Then (1.1) possesses infinitely many bounded solutions.

For a nonexistence result, Cheng and Lin [CL, Theorem 5.3] improved Oleinik's result. They proved

Theorem D. Suppose that $K \leq 0$ on $\mathbb{R}^{n}$ and $|K(x)| \geq C|x|^{-2}(\log |x|)^{-1}$ for $|x|$ large for some constant $C>0$. Then (1.1) possesses no entire solution.

From the results of Theorems $\mathrm{C}$ and $\mathrm{D}$, it seems natural to conjecture that if $K \leq 0$ on $\mathbb{R}^{n},|K(x)| \geq \widetilde{K}(|x|)$ and $\int_{0}^{\infty} t \tilde{K}(t) d t=\infty$, then (1.1) possesses no entire solution. Interestingly enough, this conjecture is not true. Lin and Cheng [LC] constructed a solution for equation (1.1) with $K=K(|x|) \leq 0$ satisfying

$$
\int_{0}^{\infty} t K(t) d t=-\infty
$$

If we try to investigate the structure of the set of solutions for equation (1.1), then Theorems $\mathrm{B}$ and $\mathrm{C}$ are not enough. Thus along this direction, we try first to find the type of solution $u_{c}$ satisfying (1.2). The following theorem answers parts of the existence question of the type of solution $u_{c}$.

Theorem 1.1. Suppose that $K$ satisfies one of the following conditions:

(i) $|x| \leq \widetilde{K}(|x|)$ on $\mathbb{R}^{n}$ and $\int_{0}^{\infty} t \widetilde{K}(t) d t<\infty$,

(ii) $\int_{\mathbb{R}^{n}}|K(y)| /|y|^{n-2} d y<\infty$ and

$$
M(x)|x|^{2}(g(x))^{2 /(n-2)} \rightarrow 0 \quad \text { as }|x| \rightarrow \infty,
$$


where

$$
M(x)=\sup _{|y-x|<|x| / 2}|K(y)|
$$

and

$$
g(x)=\int_{|y-x|<|x| / 2} \frac{|K(y)|}{|y|^{n-2}} d y .
$$

Then we have

$(\alpha)$ There exists a constant $c_{0} \in \mathbb{R}$, such that for every $c \in\left(-\infty, c_{0}\right],(1.1)$ possesses a solution $u_{c}$ satisfying (1.2).

( $\beta$ ) If, in addition, $K \leq 0$ on $\mathbb{R}^{n}$, then for every $c \in(-\infty, \infty),(1.1)$ possesses a unique solution $u_{c}$ satisfying (1.2).

( $\gamma$ ) If, in addition, $K \geq 0$ on $\mathbb{R}^{n}$, then there exists a constant $c_{0} \in \mathbb{R}$, such that for every $c \in\left(-\infty, c_{0}\right),(1.1)$ possesses a solution $u_{c}$ satisfying (1.2) and (1.1) possesses no solution $u_{c}$ satisfying (1.2) for $c>c_{0}$.

( $\delta)$ Let $u$ be a bounded solution of (1.1). Then there exists a constant $c \in \mathbb{R}$ such that $u=u_{c}$, that is, $u(x) \rightarrow c$ as $|x| \rightarrow \infty$.

A few words about this long theorem are in order. First of all, we do not divide $\mathbb{R}^{n}$ into $\mathbb{R}^{m} \times \mathbb{R}^{n-m}$ as in Theorems $\mathrm{B}$ and $\mathrm{C}$. But from the proof of Theorem (1.1) in $\S 3$ it is easy to see that some of the results can be formulated in this form. Next, we note that condition (i) is the same as in Theorem C. But even in this case, our conclusions are much more clear and complete than that of Theorem C. Finally, we need to give some explanation about condition (ii). From a slightly deep inspection of condition (i), it would be very nice if the condition

$$
\int_{\mathbb{R}^{n}} \frac{|K(y)|}{|y|^{n-2}} d y<\infty
$$

could imply the existence of the type of solution $u_{c}$. Unfortunately, this is not necessarily true. Our proof can not be carried out if $K$ concentrates on a sequence of points which tends to infinity. The other condition in (ii),

$$
M(x)|x|^{2}(g(x))^{2 /(n-2)} \rightarrow 0 \quad \text { as }|x| \rightarrow \infty,
$$

is a way to prevent such a concentration of $K$. On the other hand, in condition (i), since $|K(x)| \leq \widetilde{K}(|x|), K$ can concentrate only on a sequence of spheres, not on a sequence of points. This "spread" of $K$ is enough to guarantee the existence of solution $u_{c}$.

Are the solutions of (1.1) all of the type $u_{c}$ ? The answer is in general no. For some $K$, there is another type of solution $U$, the "maximal solution." The following theorem gives a fairly complete answer to the existence question of the maximal solution $U$ of (1.1).

Theorem 1.2. Suppose that

(i) $K \leq 0$ on $\mathbb{R}^{n}$ and there exists a sequence of bounded smooth simply connected domains $\left\{\Omega_{i}\right\}$ such that $\mathbb{R}^{n}=\bigcup_{i=1}^{\infty} \boldsymbol{\Omega}_{i}, \bar{\Omega}_{i} \subset \boldsymbol{\Omega}_{i+1}$, and $K<0$ on $\partial \Omega_{i}, i=1,2, \ldots$, and

(ii) equation (1.1) possess a solution, say $v$, on $\mathbb{R}^{n}$. 
Then the function $U(x)$ (the maximal solution) defined by (1.3) is well defined on $\mathbb{R}^{n}$ and is a solution of (1.1) on $\mathbb{R}^{n}$.

In view of Theorem 1.1, the conditions in Theorem 1.2 are quite mild. For example, if $K \leq 0$ on $\mathbb{R}^{n}$ and $C /|x|^{p} \geq|K(x)|>0$ for $|x|$ large for some constant $p>2$, then condition (i) of Theorem 1.2 holds trivially and condition (ii) also holds due to Theorem 1.1. Is condition (i) necessary for the existence of the maximal solution $U$ ? We do not know the answer to this question. But we conjecture that if $K(x)>0$ somewhere or there exists a curve extending to infinity such that $K=0$ on this curve, then the maximal solution $U$ does not exist.

Since the type of solution $u_{c}$ has nice asymptotic behavior (1.2), we are inclined to find the asymptotic behavior of the maximal solution $U$. If we can find some explicit relations between the functions $K$ and $U$, then we can easily obtain the asymptotic behavior of $U$ from that of $K$. Unfortunately, we obtain no such explicit relation.

In the following we use the notation " $f \sim g$ at $\infty$ " to denote that "there exist two positive constants $C_{1}$ and $C_{2}$ such that $C_{1} f \geq g \geq C_{2} f$ at $\infty$."

We shall consider the following possible asymptotics for $K$ and $U$.

$$
\begin{gathered}
K(x) \sim-|x|^{-p}(\log |x|)^{-\beta} \quad \text { at } \infty, \quad p>2, \beta \in \mathbb{R}, \\
K(x) \sim-|x|^{-2}(\log |x|)^{-\beta} \quad \text { at } \infty, \quad \beta>1, \\
K(x) \sim-|x|^{-q}(\log |x|)^{p-k} \exp \left[-2(\log |x|)^{p}\right] \quad \text { at } \infty, \\
K(x) \sim-|x|^{\alpha-p} \exp \left(-2|x|^{\alpha}\right) \quad \text { at } \infty, \quad \alpha>0, \quad p \in \mathbb{R}, \quad k \in \mathbb{R}, \\
U(x)=\frac{p-2}{2} \log |x|+\frac{\beta}{2} \log (\log |x|)+O(1) \quad \text { at } \infty, \\
U(x)=\frac{\beta-1}{2} \log (\log |x|)+O(1) \quad \text { at } \infty, \\
U(x)=(\log |x|)^{p}+\frac{p-2}{2} \log |x| \\
\quad+\frac{k-1}{2} \log (\log |x|)+O(1) \quad \text { at } \infty \\
U(x)=|x|^{\alpha}+\frac{p-2}{2} \log |x|+O(1) \quad \text { at } \infty .
\end{gathered}
$$

The following theorem answers only parts of the question (b) in the beginning of this section.

Theorem 1.3. Suppose that $K \leq 0$ on $\mathbb{R}^{n}$ and has one of the asymptotic behaviors (1.9), (1.10), (1.11) and (1.12). Then the maximal solution of $U$ of (1.1) exists and has the asymptotic behaviors (1.13), (1.14), (1.15) and (1.16) respectively.

Now we come to the question (c) in the beginning of this section. In other words, can we classify all the solutions of equation (1.1)? If $K(x)>0$ somewhere, then we do not know whether the type of solution $u_{c}$ is unique or not for a given $c$ and whether the maximal solution $U$ exists or not. Thus if $K(x)>0$ 
somewhere, we are still very far from the complete classification of all solutions for equation (1.1).

If $K \leq 0$ on $\mathbb{R}^{n}$, for some $K$, we can find other types of solutions besides the two types $u_{c}$ and $U$. For example, let $K(x)=-2 n e^{-2|x|^{2}}$. Let $h(x)$ be any harmonic function satisfying $h(x) \leq \frac{1}{2}|x|^{2}$ for all $x \in \mathbb{R}^{n}$. Let $u=h+w$. Then $w$ satisfies

$$
\Delta w+K(x) e^{2 h(x)} e^{2 w}=0 .
$$

Since $K(x) e^{2 h(x)}$ satisfies any one of the two conditions in Theorem 1.1, we conclude that there exists a unique solution $w_{c}$ of (1.17) satisfying $w_{c}(x) \rightarrow c$ as $|x| \rightarrow \infty$ for every real $c$. Thus for this $K$, we have a family of solutions $u=h+w_{c}, c \in \mathbb{R}$.

If $K$ has some symmetry properties, for example, $K$ is radially symmetric, from the above example, we can not expect that all solutions of (1.1) also have the same symmetry in general. But if we consider only symmetric solutions, then maybe we can classify all symmetric solutions.

Suppose now that $K \leq 0$ on $\mathbb{R}^{n}, K$ is radially symmetric and $K$ has one of the asymptotics (1.9), (1.10), (1.11) and (1.12). Then from Theorem 1.1 , equation (1.1) possesses a unique solution $u_{c}$ satisfying (1.2) for every $c \in \mathbb{R}$. Since $K$ is radially symmetric, $u_{c}(Q x)$ is also a solution of $(1.1)$ satisfying (1.2), where $Q \in O(n)$ is a rotation of $\mathbb{R}^{n}$. From uniqueness, we have $u_{c}(Q x)=u_{c}(x)$ for any $Q \in O(n)$. Hence $u_{c}$ must be also radially symmetric. Also, from Theorem 1.2, the maximal solution $U$ exists and $U$ is radially symmetric by the same arguments as above. It is natural to ask: Are $u_{c}$ and $U$ the only radial solutions for equation (1.1)? The following theorem answers this question.

Theorem 1.4. Suppose that $K \leq 0$ on $\mathbb{R}^{n}, K$ is radially symmetric and $K$ has one of the asymptotics (1.9), (1.10), (1.11) and (1.12). Let $u$ be a radial solution of equation (1.1). Then either $u=u_{c}$ for some $c \in \mathbb{R}$ or $u=U$.

Suppose now that $K \leq 0$ and $K$ has one of the asymptotics (1.9), (1.10), (1.11) and (1.12). Then from Theorems 1.1, 1.2 and 1.3, we know that (1.1) possesses a unique solution $u_{c}$ satisfying (1.2) for every $c \in \mathbb{R}$ and that the maximal solution $U$ also exists and has the asymptotics (1.13), (1.14), (1.15) and (1.16) respectively. Are $u_{c}$ and $U$ the only solutions for (1.1)? For $K$ satisfying (1.9) and (1.10), the answer is yes.

Theorem 1.5. Suppose that $K \leq 0$ on $\mathbb{R}^{n}$ and $K$ has one of the asymptotics (1.9) and (1.10). Let $u$ be a solution of (1.1). Then either $u=u_{c}$ for some $c \in \mathbb{R}$ or $u=U$.

If $K$ decays fast, for example, $K(x)=-2 n e^{-2|x|^{2}}$, then as we already noted, equation (1.1) possesses another family of solutions $u=h+w_{c}$. But we know that if $h$ is a nonconstant harmonic function on $\mathbb{R}^{n}$, then $h$ has no lower bound. Thus if we only consider solutions which have a lower bound, then maybe $u_{c}$ and $U$ are again the only solutions. We have

Theorem 1.6. Suppose that $K \leq 0$ on $\mathbb{R}^{n}$ and $K$ has one of the asymptotics (1.11) and (1.12). Let $u$ be a solution of (1.1) with a lower bound. Then either $u=u_{c}$ for some $c \in \mathbb{R}$ or $u=U$. 
Finally, we shall consider the case $K$ has compact support. The following theorem is interesting.

Theorem 1.7. Suppose that $K \leq 0$ on $\mathbb{R}^{n}$ and $K$ has compact support. Then we have

(i) For every harmonic function $h$ on $\mathbb{R}^{n}$ and every constant $c \in \mathbb{R}$, (1.1) possesses a unique solution satisfying

$$
u(x)-h(x)-c \rightarrow 0 \quad \text { as }|x| \rightarrow \infty .
$$

(ii) Let $u$ be a solution of (1.1). Then there exist a harmonic function $h$ and a constant $c$ such that $u$ satisfies (1.18).

Our main method of proofs is similar to that of Cheng and Ni [CN1, CN2]. For more background material, we refer the reader to $\mathrm{Ni}$ [N1, N2].

The organization of this paper is as follows. In $\S 2$, we shall sketch the proof of Theorem 1.2. We do not give a complete proof of Theorem 1.2 here because it is almost the same as in Cheng and $\mathrm{Ni}$ [CN1]. In $\S 3$, we shall give a detailed proof of Theorem 1.1. We shall prove Theorems 1.5, 1.6 and 1.7 in $\S 5$.

We would like to thank the referee for his constructive suggestion about the exposition of this paper.

\section{EXISTENCE OF MAXIMAL SOlUtions}

We refer the reader to Cheng and Ni [CN1] for details of the proof. We only sketch the proof here.

Sketch of the proof of Theorem 1.2. First we consider the following boundary value problem

$$
\begin{cases}\Delta u+K e^{2 u}=0 & \text { in } \Omega_{i}, \\ u(x) \rightarrow \infty & \text { as } x \rightarrow \partial \Omega_{i} .\end{cases}
$$

We use barrier method to prove the existence of a solution $u_{i}$ of $(2.1)$ for each $i=1,2, \ldots$. Here the assumptions (i) and (ii) of Theorem 1.2 are crucial. By the maximum principle, we prove that $\left\{u_{i}\right\}$ is a monotonically decreasing sequence of functions. Hence by usual elliptic regularity arguments, we prove that $u_{i}$ converges monotonically to a solution $U$ of (1.2) which is the maximal solution. Q.E.D.

We also list some important and useful properties of the maximal solutions here. We refer the reader to Cheng and Ni [CN1] for details of the proofs.

Theorem 2.1. Suppose that the hypothesis of Theorem 1.2 hold and that $\widetilde{K} \leq K \leq$ 0 on $\mathbb{R}^{n}$. Let $U$ ( $\widetilde{U}$ respectively) be the maximal solution of $\Delta u+K e^{2 u}=0$ $\left(\Delta u+\widetilde{K} e^{2 u}=0\right.$ respectively) on $\mathbb{R}^{n}$. Then $\widetilde{U} \leq U$.

Theorem 2.2. Suppose that $K$ is radially symmetric. Then the maximal solution $U$ (if exists) is also radially symmetric.

Theorem 2.3. Suppose that $K \leq 0$ in $\mathbb{R}^{n}$ and that $U$ is the maximal solution of (1.1) on $\mathbb{R}^{n}$. Let $u$ be an arbitrary solution of (1.1) on $\mathbb{R}^{n}$. Then either $u<U$ everywhere on $\mathbb{R}^{n}$ or $u=U$ on $\mathbb{R}^{n}$. 


\section{EXISTENCE OF SOLUTIONS OF THE TYPE $u_{c}$}

In this section we shall give a detailed proof of Theorem 1.1. We decompose the proof into several lemmas.

Lemma 3.1. Suppose that $K$ satisfies the hypothesis (ii) of Theorem 1.1. Then the function $v$ defined by

$$
v(x)=\frac{1}{2(2-n) w_{n}} \int_{\mathbb{R}^{n}} \frac{K(y)}{|x-y|^{n-2}} d y
$$

is well defined on $\mathbb{R}^{n}$ and satisfies

$$
v(x) \rightarrow 0 \quad \text { as }|x| \rightarrow \infty
$$

and

$$
\Delta v(x)=K(x), \quad x \in \mathbb{R}^{n},
$$

where

$$
w_{n}=\int_{|y|<1} d y
$$

Proof. We write

$$
v(x)=C_{n} \int_{|y| \leq 2|x|} \frac{K(y)}{|x-y|^{n-2}} d y+C_{n} \int_{|y| \geq 2|x|} \frac{K(y)}{|x-y|^{n-2}} d y,
$$

where $C_{n}=1 / n(2-n) w_{n}$. Now

$$
\left|C_{n} \int_{|y| \geq 2|x|} \frac{|K(y)|}{|x-y|^{n-2}} d y\right| \leq\left|C_{n}\right| \int_{|y| \geq 2|x|} \frac{2^{n-2}|K(y)|}{|y|^{n-2}} d y .
$$

Hence $v$ is well defined on $\mathbb{R}^{n}$. Equation (3.3) is a consequence of (3.1) and the assumption that $K$ is Hölder continuous. Thus it remains to prove (3.2). Let $\varepsilon>0$ be given. Choose $N$ and $R_{\varepsilon}>2 N$, such that

$$
\begin{gathered}
4^{n-2} \int_{|y| \geq N} \frac{|K(y)|}{|y|^{n-2}} d y<\varepsilon \\
\frac{(2 N)^{n-2}}{|x|^{n-2}} \int_{\mathbb{R}^{n}} \frac{|K(y)|}{|y|^{n-2}} d y<\varepsilon \quad \text { for }|x|>R_{\varepsilon}, \\
\frac{n}{2}\left(\frac{3}{2}\right)^{2-4 / n} w_{n}^{1-2 / n}\left(M(x)|x|^{2} g(x)^{2 /(n-2)}\right)^{(n-2) / n}<\varepsilon, \quad|x|>R_{\varepsilon} .
\end{gathered}
$$

Then for $|x|>R_{\varepsilon}$, we have

$$
\begin{aligned}
\int \frac{|K(y)|}{|x-y|^{n-2}} d y & =\int_{|y|<|x| / 2}+\int_{\substack{|x| / 2<|y|<2|x| \\
|y-x|>|x| / 2}}+\int_{|y-x|<|x| / 2}+\int_{|y|>2|x|} \\
& =\mathrm{I}_{1}(x)+\mathrm{I}_{2}(x)+\mathrm{I}_{3}(x)+\mathrm{I}_{4}(x) .
\end{aligned}
$$


We now estimate $I_{1}, I_{2}, I_{3}$ and $I_{4}$.

$$
\begin{aligned}
& \mathrm{I}_{1}(x)=\int_{|y|<|x| / 2} \frac{|K(y)|}{|x-y|^{n-2}} d y \\
& =\left(\int_{|y|<N}+\int_{N<|y|<|x| / 2}\right) \frac{|K(y)|}{|y-x|^{n-2}} d y \\
& \leq \int_{|y|<N} \frac{2^{n-2}|K(y)|}{|x|^{n-2}} d y+\int_{N<|y|<|x| / 2} \frac{2^{n-2}|K(y)|}{|x|^{n-2}} d y \\
& \leq \frac{(2 N)^{n-2}}{|x|^{n-2}} \int_{|y|<N} \frac{|K(y)|}{|y|^{n-2}} d y+\int_{N<|y|<|x| / 2} \frac{|K(y)|}{|y|^{n-2}} d y \\
& \leq \frac{(2 N)^{n-2}}{|x|^{n-2}} \int_{\mathbb{R}^{n}} \frac{|K(y)|}{|y|^{n-2}} d y+\int_{|y|>N} \frac{|K(y)|}{|y|^{n-2}} d y \\
& <2 \varepsilon \quad \text { (from (3.4) and (3.5)) . } \\
& \mathrm{I}_{2}(x)=\int_{\substack{|x| / 2<|y|<2|x| \\
|y-x|>|x| / 2}} \frac{|K(y)|}{|x-y|^{n-2}} d y \\
& \leq \int_{\substack{|x| / 2<|y|<2|x| \\
|y-x|>|x| / 2}} \frac{2^{n-2}|K(y)|}{|x|^{n-2}} d y \\
& \leq 4^{n-2} \int_{\substack{|x| / 2<|y|<2|x| \\
|y-x|>|x| / 2}} \frac{|K(y)|}{|y|^{n-2}} d y \\
& \leq 4^{n-2} \int_{|y|>N} \frac{|K(y)|}{|y|^{n-2}} d y<\varepsilon \quad(\text { from }(3.4)) \text {. } \\
& \mathbf{I}_{4}(x)=\int_{|y|>2|x|} \frac{|K(y)|}{|x-y|^{n-2}} d y \\
& \leq 2^{n-2} \int_{|y|>2|x|} \frac{|K(y)|}{|y|^{n-2}} d y \\
& \leq 4^{n-2} \int_{|y|>N} \frac{|K(y)|}{|y|^{n-2}} d y<\varepsilon \quad(\text { from (3.4)). }
\end{aligned}
$$

Finally we estimate $\mathrm{I}_{3}(x)$. The estimate of $\mathrm{I}_{3}(x)$ is much more involved. It is here we have to use (1.8). For $\rho \in[0,|x| / 2]$, we define the function $\eta=\eta(\rho)$ by the following ordinary differential equation

$$
\left\{\begin{array}{l}
\left(\frac{\eta(\rho)}{\rho}\right)^{n-1} \frac{d \eta(\rho)}{d \rho}=\frac{1}{n w_{n}} \int_{|z|=1} \frac{1}{M(x)}|K(x+\rho z)| d S_{z}, \\
\eta(0)=0
\end{array}\right.
$$

Here we of course assume that $M(x)>0$. If $M(x)=0$, then $\mathrm{I}_{3}=0$. We do not have to estimate $I_{3}(x)$.

From (1.5), since

$$
\frac{1}{n w_{n}} \int_{|z|=1} d S_{z}=1
$$


we have

Hence we have

$$
\left(\frac{\eta(\rho)}{\rho}\right)^{n-1} \frac{d \eta(\rho)}{d \rho} \leq 1, \quad 0 \leq \rho \leq \frac{|x|}{2}
$$

$$
\eta(\rho) \leq \rho \quad \text { for } 0 \leq \rho \leq \frac{|x|}{2}
$$

Let

$$
\varepsilon(x)=\eta\left(\frac{|x|}{2}\right)
$$

Then from (3.7) we have

$$
\begin{aligned}
\frac{1}{n}(\varepsilon(x))^{n} & =\int_{0}^{\varepsilon(x)} \eta^{n-1} d \eta \\
& =\int_{0}^{|x| / 2} \rho^{n-1}\left[\frac{1}{n w_{n}} \int_{|z|=1} \frac{1}{M(x)}|K(x+\rho z)| d S_{z}\right] d \rho \\
& =\frac{1}{n w_{n} M(x)} \int_{|y-x|<|x| / 2}|K(y)| d y \\
& \leq \frac{1}{n w_{n} M(x)}\left(\frac{3}{2}|x|\right)^{n-2} g(x) .
\end{aligned}
$$

Now we can estimate $\mathrm{I}_{3}(x)$.

$$
\begin{aligned}
\mathrm{I}_{3}(x) & =\int_{|y-x|<|x| / 2} \frac{|K(y)|}{|y-x|^{n-2}} d y \\
& =\int_{0}^{|x| / 2} \rho^{n-1}\left[\rho^{2-n} \int_{|z|=1}|K(x+\rho z)| d S_{z}\right] d \rho \\
& =n w_{n} M(x) \int_{0}^{\varepsilon(x)} \rho^{2-n} \eta^{n-1} d \eta \quad(\text { from }(3.7)) \\
& \leq n w_{n} M(x) \int_{0}^{\varepsilon(x)} \eta d \eta \quad(\text { from }(3.8)) \\
& =\frac{n}{2} w_{n} M(x)(\varepsilon(x))^{2} \\
& \leq \frac{n}{2}\left(\frac{3}{2}\right)^{2-4 / n} w_{n}^{1-2 / n}\left[M(x)|x|^{2} g(x)^{2 /(n-2)}\right]^{(n-2) / n} \\
& <\varepsilon \quad(\text { from }(3.6)) .
\end{aligned}
$$

Hence we have

$$
\int_{\mathbb{R}^{n}} \frac{|K(y)|}{|y-x|^{n-2}} d y=\mathrm{I}_{1}(x)+\mathrm{I}_{2}(x)+\mathrm{I}_{3}(x)+\mathrm{I}_{4}(x) \leq 5 \varepsilon
$$

for $|x|>R_{\varepsilon}$. Since $\varepsilon>0$ is arbitrary, this proves that

$$
\int_{\mathbb{R}^{n}} \frac{|K(y)|}{|y-x|^{n-2}} d y \rightarrow 0 \quad \text { as }|x| \rightarrow \infty .
$$

Thus (3.2) is proved. Q.E.D. 
Lemma 3.2. Suppose that $\widetilde{K}=\widetilde{K}(r) \geq 0$ on $0 \leq r<\infty$ and satisfies $\int_{0}^{\infty} r \widetilde{K} d r<$ $\infty$. Then the function

$$
v(x)=\frac{1}{n(2-n) w_{n}} \int_{\mathbb{R}^{n}} \frac{\tilde{K}(|y|)}{|y-x|^{n-2}} d y
$$

is well defined on $\mathbb{R}^{n}$ and satisfies

$$
v(x) \rightarrow 0 \quad \text { as }|x| \rightarrow \infty
$$

and

$$
\Delta v(x)=\widetilde{K}(|x|) \quad \text { on } \mathbb{R}^{n} .
$$

Proof. From the proof of Lemma 3.1, the only thing we have to check is

$$
\mathrm{I}_{3}(x)=\int_{|y-x|<|x| / 2} \frac{\widetilde{K}(|y|)}{|x-y|^{n-2}} d y \rightarrow 0 \quad \text { as }|x| \rightarrow \infty .
$$

We use spherical coordinates. Let $x=|x| z_{0}$ and $y=|y| z$, where $|z|=1=$ $\left|z_{0}\right|$. Let $|y|=r$. Then

$$
\begin{aligned}
\mathrm{I}_{3}(x) & =\int_{|y-x|<|x| / 2} \frac{\widetilde{K}(|y|)}{|x-y|^{n-2}} d y \\
& =\int_{|x| / 2}^{\frac{3}{2}|x|} r^{n-1} \widetilde{K}(r)\left[\int_{|z| \in \Phi(r)} \frac{1}{|r z-| x\left|z_{0}\right|^{n-2}} d S_{z}\right] d r,
\end{aligned}
$$

where $\Phi(r) \subseteq\{z|| z \mid=1\}$. it is not difficult to prove that there exists a constant $C>0$ such that for $|y-x|<|x| / 2$,

$$
|y-x|=|r z-| x\left|z_{0}\right| \geq C|| x|z-| x\left|z_{0}\right|=C|x|\left|z-z_{0}\right| .
$$

Hence,

$$
\begin{aligned}
C^{n-2} \mathrm{I}_{3}(x) & \leq \int_{|x| / 2}^{\frac{3}{2}|x|} r^{n-1} \widetilde{K}(r)\left[\int_{z \in \Phi(r)} \frac{d S_{z}}{|x|^{n-2}\left|z-z_{0}\right|^{n-2}}\right] d r \\
& \leq\left(\int_{|x| / 2}^{\frac{3}{2}|x|} r^{n-1} \widetilde{K}(r) \frac{d r}{|x|^{n-2}}\right)\left(\int_{|z|=1} \frac{d S_{z}}{\left|z-z_{0}\right|^{n-2}}\right) \\
& \leq\left(\frac{3}{2}\right)^{n-2}\left(\int_{|x| / 2}^{\infty} r \widetilde{K}(r) d r\right)\left(\int_{|z|=1} \frac{d S_{z}}{\left|z-z_{0}\right|^{n-2}}\right)
\end{aligned}
$$

Now,

$$
\int_{|z|=1} \frac{d S_{z}}{\left|z-z_{0}\right|^{n-2}}
$$

is a finite number and

$$
\int_{|x| / 2}^{\infty} r \widetilde{K}(r) d r \rightarrow 0 \quad \text { as }|x| \rightarrow \infty .
$$

Thus $\mathrm{I}_{3}(x) \rightarrow 0$ as $|x| \rightarrow \infty$. Q.E.D.

Lemma 3.3. Suppose that $K_{1} \geq K_{2} \geq 0$ on $\mathbb{R}^{n}$ and $K_{1}$ satisfies the hypotheses in Theorem 1.1. Suppose that the equation $\Delta u+K_{1} e^{2 u}=0$ has a solution $u$ 
satisfying $u(x) \rightarrow c$ as $|x| \rightarrow \infty$. Then the equation $\Delta v+K_{2} e^{2 v}=0$ also possesses a solution $v$ satisfying $v(x) \rightarrow c$ as $|x| \rightarrow \infty$.

Proof. Since $u(x) \rightarrow c$ as $|x| \rightarrow \infty, u$ is a bounded super harmonic function. On the other hand, $K_{1}$ satisfies the hypotheses in Theorem 1.1. Hence

$$
\int_{\mathbb{R}^{n}} \frac{1}{|x|^{n-2}} K_{1}(x) e^{2 u(x)} d x<\infty .
$$

From [HK, Theorem 3.20], we have

$$
u(x)=c-C_{n} \int_{\mathbb{R}^{n}} \frac{1}{|x-y|^{n-2}} K_{1}(y) e^{2 u(y)} d y,
$$

where $C_{n}=1 / n(2-n) w_{n}$. Let $u_{0} \equiv c$ and

$$
u_{m+1}=c-C_{n} \int_{\mathbb{R}^{n}} \frac{1}{|x-y|^{n-2}} K_{1}(y) e^{2 u_{m}(y)} d y, \quad m=0,1,2, \ldots
$$

Then it is easy to see that

$$
u_{m+1} \geq u_{m} \text { on } \mathbb{R}^{n}, \quad m=0,1,2, \ldots,
$$

and

$$
u_{m} \leq u \text { on } \mathbb{R}^{n}, \quad m=0,1,2, \ldots
$$

Now let $v_{0} \equiv c$ and

$$
v_{m+1}=c-C_{n} \int_{\mathbb{R}^{n}} \frac{1}{|x-y|^{n-2}} K_{2}(y) e^{2 v_{m}(y)} d y, \quad m=0,1,2, \ldots .
$$

From the assumption $K_{1} \geq K_{2} \geq 0$ on $\mathbb{R}^{n}$, we have

$$
u_{m} \geq v_{m} \text { on } \mathbb{R}^{n} \quad \text { for all } m=0,1,2, \ldots \text {. }
$$

and

$$
v_{m+1} \geq v_{m} \text { on } \mathbb{R}^{n}, \quad m=0,1,2, \ldots .
$$

Hence $\left\{v_{m}\right\}$ is a monotonically increasing sequence of functions which is bounded by $u$ on $\mathbb{R}^{n}$. Hence $\left\{v_{m}\right\}$ converges to a function $v$ on $\mathbb{R}^{n}$ and $v$ satisfies

$$
v(x)=c-C_{n} \int_{\mathbb{R}^{n}} \frac{1}{|x-y|^{n-2}} K_{2}(y) e^{2 v(y)} d y .
$$

This proves that $v$ is a solution of the equation $\Delta u+K_{2} e^{2 v}=0$ on $\mathbb{R}^{n}$ and $v(x) \rightarrow c$ as $|x| \rightarrow \infty$. Q.E.D.

Lemma 3.4. Let

$$
v(r)=\gamma-\frac{1}{2} \log \left(1+\alpha r^{2}\right), \quad r \geq 0
$$

Then $v$ satisfies

$$
v^{\prime \prime}(r)+\frac{n-1}{r} v^{\prime}(r)+\bar{K}(r) e^{2 v}=0,
$$

where $\bar{K}(r)=e^{-2 \gamma} \alpha\left[(n-2)+2\left(1+\alpha r^{2}\right)^{-1}\right]$.

Proof. Direct calculation yields the lemma. Q.E.D. 
Lemma 3.5. Suppose that $K \geq 0$ on $\mathbb{R}^{n}$ and that (1.1) has a solution $u_{0}$ with $u_{0}(x) \rightarrow c_{0}$ as $|x| \rightarrow \infty$, for some $c_{0} \in \mathbb{R}$. Then for every $c<c_{0}$, (1.1) possesses a solution with $u(x) \rightarrow c$ as $|x| \rightarrow \infty$.

Proof. $u_{0}$ is a superharmonic function with $u_{0}(x) \rightarrow c_{0}$ as $|x| \rightarrow \infty$. By the maximum principle, $u_{0}(x) \geq c_{0}$ for all $x \in \mathbb{R}^{n}$. Let $c<c_{0}, u_{-}(x)=c$ and $u_{+}(x)=u_{0}(x)-\left(c_{0}-c\right)$. Then

$$
\begin{aligned}
\Delta u_{+}+K e^{2 u_{+}} & =\Delta u_{0}+K e^{2\left(u_{0}-c_{0}\right)+2 c} \\
& =-K e^{2 u_{0}}+K e^{2 u_{0}-2\left(c_{0}-c\right)} \\
& =K e^{2 u_{0}}\left(-1+e^{-2\left(c_{0}-c\right)}\right) \leq 0,
\end{aligned}
$$

and

$$
\Delta u_{-}+K e^{2 u_{-}}=K e^{2 c} \geq 0 .
$$

Hence $u_{+}$and $u_{-}$are respectively a supersolution and subsolution of (1.1) satisfying $u_{+} \geq u_{-}$on $\mathbb{R}^{n}$. Hence there exists a solution $u$ of (1.1) satisfying $u_{+} \geq u \geq u_{-}$on $\mathbb{R}^{n}$. Since $u_{+}(x) \rightarrow c$ and $u_{-}(x) \rightarrow c$ as $|x| \rightarrow \infty$, we conclude that $u(x) \rightarrow c$ as $|x| \rightarrow \infty$. Q.E.D.

Lemma 3.6. Suppose that $K \geq 0$ on $\mathbb{R}^{n}$ and that (1.1) possesses a solution $u$ with $u(x) \rightarrow c$ as $|x| \rightarrow \infty$. Then there exists a constant $c_{0}$, depending only on $K$, such that $c \leq c_{0}$.

Proof. Without loss of generality, we assume that $K(0)>0$. Since $K$ is locally Hölder continuous, there exist constants $\varepsilon>0$ and $\delta>0$ such that

$$
K(x) \geq \varepsilon \quad \text { for }|x| \leq \delta .
$$

Now choose $\alpha_{0}>0$ and $\eta>0$ such that

$$
K_{\alpha}(r)=\eta\left[(n-2)+2\left(1+\alpha r^{2}\right)^{-1}\right] \leq \varepsilon
$$

for all $\alpha \geq \alpha_{0}$ and $r \in[0, \delta]$. Let

$$
e^{-2 \gamma} \cdot \alpha=\eta, \quad \alpha \geq \alpha_{0} .
$$

Consider the following ordinary differential equation

$$
\begin{cases}u^{\prime \prime}+\frac{n-1}{r} u^{\prime}+K_{\alpha}(r) e^{2 u}=0, & 0<r<\delta, \\ u^{\prime \prime}+\frac{n-1}{r} u^{\prime}=0, & \delta \leq r<\infty, \\ u(0)=\gamma, \quad u^{\prime}(0)=0, & \alpha \geq \alpha_{0} .\end{cases}
$$

From Lemma 3.4, the solution $u_{\alpha}$ of (3.24) is

$$
u_{\alpha}(r)=\left\{\begin{array}{rr}
\gamma-\frac{1}{2} \log \left(1+\alpha r^{2}\right) \quad \text { for } 0 \leq r<\delta, \\
\gamma-\frac{1}{2} \log \left(1+\alpha \delta^{2}\right)-\frac{1}{n-2} \alpha \delta^{n}\left(1+\alpha \delta^{2}\right)^{-1}\left(\delta^{2-n}-r^{2-n}\right) \\
\text { for } \delta \leq r<\infty
\end{array}\right.
$$

Hence

$$
u_{\alpha}(\infty)=\lim _{r \rightarrow \infty} u_{\alpha}(r)=\gamma-\frac{1}{2} \log \left(1+\alpha \delta^{2}\right)-\frac{1}{n-2} \alpha \delta^{2}\left(1+\alpha \delta^{2}\right)^{-1} .
$$

Using (3.23), we have

$$
u_{\alpha}(\infty)=\frac{1}{2} \log \left(\frac{\alpha}{\eta}\right)-\frac{1}{2} \log \left(1+\alpha \delta^{2}\right)-\frac{1}{n-2} \alpha \delta^{2}\left(1+\alpha \delta^{2}\right)^{-1} .
$$


Let

$$
c_{0}=\max \left\{a_{0}, \sup _{\alpha \geq \alpha_{0}} u_{\alpha}(\infty)\right\} .
$$

Then from Lemma 3.3, we conclude that $c \leq c_{0}$. Q.E.D.

Now we are in a position to prove Theorem 1.1 .

Proof of Theorem 1.1. First we shall prove that there exists a function $v$ satisfying

$$
\begin{gathered}
\Delta v=|K| \quad \text { on } \mathbb{R}^{n}, \\
v(x) \rightarrow 0 \quad \text { as }|x| \rightarrow \infty .
\end{gathered}
$$

But from Lemma 3.1, the existence of $v$ is assured if $K$ satisfies the hypothese (ii). Thus it remains to prove (3.28) and (3.29) if $K$ satisfies the hypothese (i). But from Lemma 3.2, there exists a function $\tilde{v}$, such that, $\Delta \tilde{v}(x)=\widetilde{K}(|x|)$ and $\tilde{v}(x) \rightarrow 0$ as $|x| \rightarrow \infty$. From the maximum principle, $\tilde{v}(x) \leq 0$ for all $r \in \mathbb{R}^{n}$. Thus

$$
\Delta \tilde{v}(x)-|K(x)|=\tilde{K}(|x|)-|K(x)| \geq 0 .
$$

Hence $\tilde{v}$ and 0 are respectively a subsolution and a supersolution of (3.28). Thus there exists a function $v$ satisfying (3.28) and (3.29). Thus under the hypotheses of Theorem 1.1, we can find a function $v$ satisfying (3.28) and (3.29). By the maximum principle, $v(x) \leq 0$ on $\mathbb{R}^{n}$. Let

$$
c_{0}=\inf _{x \in \mathbb{R}^{n}} v(x) \text {. }
$$

For $c \in\left(-\infty, c_{0}\right]$, let $u_{+}=-v+c$ and $u_{-}=\beta v+c, \beta \geq e^{2 c}$. Then we have

$$
\begin{aligned}
\Delta u_{+}+K e^{2 u_{+}} & =-\Delta v+K e^{-2 v+2 c}=-|K|+K e^{-2 v} e^{2 c} \\
& \leq|K|\left[-1+e^{2\left(c-c_{0}\right)}\right] \leq 0
\end{aligned}
$$

and

$$
\begin{aligned}
\Delta u_{-}+K e^{2 u_{-}} & =\beta|K|+K e^{2 c} e^{2 \beta v} \geq \beta|K|-|K| e^{2 c} e^{2 \beta v} \\
& \geq|K|\left(\beta-e^{2 c}\right) \geq 0 .
\end{aligned}
$$

Since $u_{+} \geq u_{-}, u_{+}(x) \rightarrow c$ as $|x| \rightarrow \infty$ and $u_{-}(x) \rightarrow c$ as $|x| \rightarrow \infty$, we conclude that there exists a solution $u_{c}$ of (1.1) satisfying $u_{+} \geq u_{c} \geq u_{-}$and hence $u_{c}(x) \rightarrow c$ as $|x| \rightarrow \infty$. This proves $(\alpha)$.

Now suppose that in addition $K \leq 0$ on $\mathbb{R}^{n}$. Then as above, there exists a function $v$ satisfying

$$
\Delta v=-K, \quad v \leq 0 \quad \text { on } \mathbb{R}^{n}
$$

and

$$
v(x) \rightarrow 0 \quad \text { as }|x| \rightarrow \infty \text {. }
$$

Let $c \in(-\infty, \infty)$ be a given number. Let $u_{+}(x)=c$ and $u_{-}(x)=\alpha v+c$ with $\alpha>e^{2 c}$. Then $u_{+} \geq u_{-}$on $\mathbb{R}^{n}$ and

$$
\begin{aligned}
\Delta u_{+}+K e^{2 u_{+}} & =K e^{2 c} \leq 0, \\
\Delta u_{-}+K e^{2 u_{-}} & =\alpha(-K)+K e^{2 \alpha v+2 c} \geq(-K)\left(\alpha-e^{2 c}\right) \geq 0 .
\end{aligned}
$$


Thus there exists a solution $u_{c}$ of (1.1) satisfying $u_{+} \geq u_{c} \geq u_{-}$and $u_{c}(x) \rightarrow c$ as $|x| \rightarrow \infty$. We shall leave the proof of uniqueness of $u_{c}$ to a general theorem in $\S 4$. This proves $(\beta)$.

Suppose now that $K \geq 0$ on $\mathbb{R}^{n}$. Then from part $(\alpha)$, there exists a constant $c_{1}$, such that, for every $c \in\left(-\infty, c_{1}\right],(1.1)$ possesses a solution $u_{c}$ with $u_{c}(x) \rightarrow c$ as $|x| \rightarrow \infty$. Let

$A=\left\{c \mid(1.1)\right.$ possesses a solution $u_{c}$ with $u_{c}(x) \rightarrow c$ as $\left.|x| \rightarrow \infty\right\}$.

Then $\left(-\infty, c_{1}\right] \subset A$. From Lemma 3.6, $A$ is bounded from above. Let $c_{0}=$ $\sup A$. Then from Lemma 3.5, we have $A=\left(-\infty, c_{0}\right]$ or $A=\left(-\infty, c_{0}\right)$. This proves $(\gamma)$.

Finally let $u$ be a bounded solution of (1.1). As in the beginning of this proof, there exists a function $v$ satisfying $\Delta v=-K e^{2 u}$ on $\mathbb{R}^{n}$ and $v(x) \rightarrow 0$ as $|x| \rightarrow \infty$. Let $w=u-v$. We have $\Delta w=0$. Hence $w$ is a bounded harmonic function on $\mathbb{R}^{n}$. Hence $w \equiv$ constant $=c$ for some $c \in \mathbb{R}$. This proves $(\delta)$. Q.E.D.

\section{Proofs of Theorems 1.3 AND 1.4}

In this section we shall prove Theorems 1.3 and 1.4. But first we give a uniqueness theorem as promised in the proof of Theorem 1.1( $\beta)$ in $\S 3$.

Theorem 4.1. Suppose that $K \leq 0$ on $\mathbb{R}^{n}$. Let $u$ and $v$ be two solutions of (1.1) satisfying $|u(x)-v(x)| \rightarrow 0$ as $|x| \rightarrow \infty$. Then $u=v$ on $\mathbb{R}^{n}$.

Proof. Let $w=(u-v)^{2}$. Then

$$
\begin{aligned}
\Delta w & =2 D(u-v) \cdot D(u-v)+2(u-v)(\Delta u-\Delta v) \\
& \geq 2(u-v)(-K)\left(e^{2 u}-e^{2 v}\right) \geq 0 .
\end{aligned}
$$

Thus $w$ is a nonnegative subharmonic function on $\mathbb{R}^{n}$ satisfying $w(x) \rightarrow 0$ as $|x| \rightarrow \infty$. Hence $w=0$ on $\mathbb{R}^{n}$. Q.E.D.

Now we prove Theorem 1.3 first.

Proof of Theorem 1.3. Suppose that $K$ satisfies equation (1.9). Then we can find $K_{1}=K_{1}(|x|)$ such that $K(x) \leq K_{1}(|x|) \leq 0$ for all $x \in \mathbb{R}^{n}$ and $K_{1}(r)=$ $-C_{1} r^{-p}(\log r)^{-\beta}$ for $r \geq R_{0}$ for some large $R_{0}$. Let $U_{1}$ be the maximal solution of $\Delta u+K_{1} e^{2 u}=0$ on $\mathbb{R}^{n}$. Then from Theorems 1.1 and 1.2, $U_{1}$ exists and $U \leq U_{1}$ on $\mathbb{R}^{n}$ from Theorem 2.1. Furthermore, $U_{1}$ is a radial function from Theorem 2.2.

So now let

$$
U_{1}(r)=w(r)+\frac{p-2}{2} \log r+\frac{\beta}{2} \log \log r \quad \text { for } r \geq R_{0} .
$$

Then $w$ satisfies

$$
\begin{aligned}
w^{\prime \prime}+ & \frac{n-1}{r} w^{\prime} \\
& =C_{1} r^{-2}\left[e^{2 w}-C_{1}^{-1}\left(\frac{(p-2)(n-2)}{2}+\frac{\beta(n-2)}{2 \log r}-\frac{\beta}{2(\log r)^{2}}\right)\right] .
\end{aligned}
$$

We can choose $R_{0}$ so large that

$$
C_{2} \geq \frac{(p-2)(n-2)}{2}+\frac{\beta}{2} \frac{n-2}{\log r}-\frac{\beta}{2(\log r)^{2}} \geq C_{3}>0,
$$


for $r \geq R_{0}$ for some constants $C_{2}$ and $C_{3}$. Let $w_{1}=\frac{1}{2} \log \left(C_{2} / C_{1}\right)$. We shall prove that there exists a constant $R_{1} \geq R_{0}$, such that, $w(r) \leq w_{1}+1$ for all $r \geq R_{1}$. Suppose that this is not true. Then either (a) $w(r) \geq w_{1}+1$ and $w^{\prime}(r)<0$ for all $r \geq R_{0}$ or (b) there exists $R_{2} \geq R_{0}$ such that $w\left(R_{2}\right) \geq w_{1}+1$ and $w^{\prime}\left(R_{2}\right) \geq 0$. If (a) holds, then from (4.1), we have

$$
\left(r^{n-1} w^{\prime}(r)\right)^{\prime} \geq \frac{C_{1}}{r^{2}} r^{n-1}\left[e^{2\left(w_{1}+1\right)}-e^{2 w_{1}}\right] .
$$

Hence we have

$$
r^{n-1} w^{\prime}(r) \geq R_{0}^{n-1} w^{\prime}\left(R_{0}\right)+C_{1}\left[e^{2 w_{1}}\left(e^{2}-1\right)\right] \frac{1}{n-2}\left(r^{n-2}-R_{0}^{n-2}\right) .
$$

Thus

$$
w^{\prime}(r) \geq C_{4} r^{-1}+O(1) r^{-(n-1)}
$$

for some $c_{4}>0$. This contradicts to the fact that $w^{\prime}(r)<0$ for all $r \geq R_{0}$. Hence (a) does not hold.

Suppose that (b) holds. Then for $r \geq R_{2}$ and $\left(r-R_{2}\right)$ small, we have $w(r) \geq w_{1}+\frac{1}{2}$ and hence

$$
\left(r^{n-1} w^{\prime}(r)\right)^{\prime} \geq C_{1} r^{n-3}\left[e^{2\left(w_{1}+\frac{1}{2}\right)}-e^{2 w_{1}}\right]=C_{5} r^{n-3}>0
$$

Hence

$$
w^{\prime}(r) \geq w^{\prime}\left(R_{2}\right) R_{2}^{n-1} r^{-(n-1)}+C_{6}\left(r^{n-2}-R_{2}^{n-2}\right) r^{-(n-1)}>0 .
$$

This proves that $w^{\prime}(r)>0$ for $r>R_{2}$ and $\left(r-R_{2}\right)$ small. Hence $w^{\prime}(r)>0$ for all $r>R_{2}$ and $w(r) \geq w_{1}+1$ for all $r \geq R_{2}$. From (4.1), we have

$$
\left[\begin{array}{l}
w^{\prime \prime}+\frac{n-1}{r} w^{\prime} \geq \frac{C_{7}}{r^{2}} e^{2 w}, \quad r>R_{2}, \\
w\left(R_{2}\right) \geq w_{1}+1, \quad w^{\prime}\left(R_{2}\right) \geq 0 .
\end{array}\right.
$$

From Cheng and Lin [CL], (4.3) possesses no solution $w$ on $\left[R_{2}, \infty\right)$. Hence (b) does not hold also. Thus $w(r) \leq w_{1}+1$ for $r$ large. This proves that

$$
U(x) \leq U_{1}(|x|) \leq \frac{p-2}{2} \log |x|+\frac{\beta}{2} \log \log |x|+C
$$

for $|x|$ large for some $C$.

Now let

$$
U_{2}(r)=\frac{p-2}{4} \log \left(1+r^{2}\right)+\frac{\beta}{2} \log \log \left(a+r^{2}\right)-b,
$$

where $a$ and $b$ are two positive constants to be determined later. $U_{2}(|x|)$ satisfies

$$
\Delta U_{2}+K_{2} e^{2 U_{2}}=0 \quad \text { on } \mathbb{R}^{n},
$$

where $K_{2}(r)=-F(r) G(r)$ and

$$
\begin{gathered}
F(r)=\left(1+r^{2}\right)^{-(p-2) / 2}\left[\log \left(a+r^{2}\right)\right]^{-\beta} e^{2 b} \\
G(r)=\left\{\frac{p-2}{2} \frac{n+(n-2) r^{2}}{\left(1+r^{2}\right)^{2}}+\beta\left[\frac{\left(n+(n-2) r^{2}\right) \log \left(a+r^{2}\right)-2 r^{2}}{\left(a+r^{2}\right)^{2}\left(\log \left(a+r^{2}\right)\right)^{2}}\right]\right\} .
\end{gathered}
$$


It is easy to see that $K_{2}(r) \sim-r^{-p}(\log r)^{-\beta}$ at $\infty$. We choose $a$ so large that $K_{2}(r)<0$ for all $r \geq 0$ and then choose $b$ so large that $K_{2}(|x|) \leq K(x)$ for all $x \in \mathbb{R}^{n}$. From Theorem 2.1, $U_{2}(|x|) \leq U(x)$. Thus

$$
\frac{p-2}{2} \log |x|+\frac{\beta}{2} \log \log |x|-C \leq U_{2}(x) \leq U(x)
$$

for $|x|$ large for some $C$.

Combing (4.4) and (4.6) we prove (1.13).

Now suppose that $K$ satisfies (1.10). We shall prove that $U$ satisfies (1.14). Since the method of the proof is similar to that of (1.13), we shall only indicate the corresponding choices of $K_{1}, U_{1}, K_{2}, U_{2}$. We can choose

$$
K_{1}(r)=-C_{1} r^{-2}(\log r)^{-\beta}, \quad \text { for }|x| \text { large. }
$$

Then

$$
U(x) \leq U_{1}(|x|) \leq \frac{\beta-1}{2} \log \log (|x|)+C
$$

for $|x|$ large. We choose

$$
\begin{gathered}
U_{2}(r)=\frac{\beta-1}{2} \log \log \left(a+r^{2}\right)-b, \\
K_{2}(r)=-(\beta-1)\left(a+r^{2}\right)^{-1}\left(\log \left(a+r^{2}\right)\right)^{-\beta} e^{2 b} \\
\cdot\left\{\frac{n a+(n-2) r^{2}}{a+r^{2}}-\frac{2 r^{2}}{\left(a+r^{2}\right) \log \left(a+r^{2}\right)}\right\} .
\end{gathered}
$$

We can choose $a$ so large that $K_{2}(r)<0$ for all $r \geq 0$ and choose $b$ so large that $K_{2}(|x|) \leq K(x)$ for all $x \in \mathbb{R}^{n}$. Then

$$
\frac{\beta-1}{2} \log \log |x|-C \leq U_{2}(|x|) \leq U(x) \quad \text { for }|x| \text { large. }
$$

This proves (1.14). Suppose now that $K$ satisfies (1.11). We choose

$$
K_{1}(r)=-C_{1} r^{-q}(\log r)^{p-k} e^{-2(\log r)^{p}} \quad \text { for } r \text { large. }
$$

Then

$$
U(x) \leq U_{1}(|x|) \leq(\log |x|)^{p}+\frac{q-2}{2} \log |x|+\frac{k-1}{2} \log \log |x|+C
$$

for $|x|$ large. We choose

$$
U_{2}(r)=\left\{\begin{array}{l}
a r^{2}+b r^{p+1}+c-d \quad \text { for } r \leq R_{0}, \\
(\log r)^{p}+\frac{q-2}{2} \log r+\frac{k-1}{2} \log \log r-d \quad \text { if } r \geq R_{0},
\end{array}\right.
$$

where $R_{0}$ is properly chosen, $a, b, c$, are chosen so that $U_{2}$ is a $C^{2}$-function on $(0, \infty)$. Now $U_{2}(|x|)$ satisfies $\Delta U_{2}+K_{2} e^{2 U_{2}}=0$, where $K_{2}$ is a negative function which can be calculated. Choose the constant $d$ so that $K_{2}(|x|) \leq$ $K(x)$ on $\mathbb{R}^{n}$. Thus $U_{2}(|x|) \leq U(x)$. This proves (1.15).

Finally suppose that $K$ satisfies equation (1.12). Then we choose $K_{1}(r)=$ $-C_{1} r^{\alpha-p} e^{-2 r^{\alpha}}$ for $r$ large. Then

$$
U(|x|) \leq U_{1}(|x|) \leq|x|^{\alpha}+\frac{p-2}{2} \log |x|+C \quad \text { for }|x| \text { large } .
$$


Let

$$
U_{2}(r)= \begin{cases}a r^{2}+b r^{\alpha+2}+c-d & \text { if } r \leq R_{0}, \\ r^{\alpha}+\frac{p-2}{2} \log r-d & \text { if } r \geq R_{0},\end{cases}
$$

where $a, b, c$, are chosen such that $U_{2}$ is a $C^{2}$-functions on $[0, \infty)$. Then $U_{2}(|x|)$ satisfies $\Delta U_{2}+K_{2} e^{2 U_{2}}=0$ for some negative function $K_{2}$. Choose $d$ so large that $K_{2}(|x|) \leq K(x) \quad \forall x \in \mathbb{R}^{n}$. Then $U_{2}(|x|) \leq U(x)$. This proves (1.16). Q.E.D.

Next we prove Theorem 1.4.

Proof of Theorem 1.4. Let $u=u(r)$ be a radial solution of (1.1). Then $u$ satisfies

$$
u(r)=u(0)+\frac{1}{n-2} \int_{0}^{r} s\left[1-\left(\frac{s}{r}\right)^{n-2}\right][-K(s)] e^{2 u(s)} d s .
$$

Let $U$ be the maximal solution of (1.1). Then $U=U(r)$ satisfies

$$
U(r)=U(0)+\frac{1}{n-2} \int_{0}^{r} s\left[1-\left(\frac{s}{r}\right)^{n-2}\right][-K(s)] e^{2 U(s)} d s .
$$

It is easy to see that $u(0) \leq U(0)$. If $u(0)=U(0)$, then $u=U$ on $\mathbb{R}^{n}$ by the uniqueness of ordinary differential equation satisfied by $u$. Suppose that $u(0)<U(0)$. Then $u(r)<U(r)$ and we have from (4.7) and (4.8)

$$
\begin{aligned}
u(r) & \leq u(0)+\frac{1}{n-2} \int_{0}^{r} s\left[1-\left(\frac{s}{r}\right)^{n-2}\right][-K(s)] e^{2 U(s)} d s \\
& =u(0)+U(r)-U(0) .
\end{aligned}
$$

Hence again from (4.7) and (4.8) we have

$$
\begin{aligned}
u(r) & \leq u(0)+\frac{1}{n-2} \int_{0}^{r} s\left[1-\left(\frac{s}{r}\right)^{n-2}\right][-K(s)] e^{2 U(s)-2(U(0)-u(0))} d s \\
& =u(0)+e^{-2(U(0)-u(0))}[U(r)-U(0)] \\
& \leq O(1)+e^{-2(U(0)-u(0))} U(r) .
\end{aligned}
$$

From Theorem 1.3, we know the asymptotic behaviors of these $U$ 's. Thus from (4.9), we can show that

$$
\int_{0}^{\infty} s[-K(s)] e^{2 u(s)} d s<\infty .
$$

From (4.7), we have $u(r) \rightarrow c$ as $r \rightarrow \infty$ for some $c$. From Theorem 4.1, we conclude that $u=u_{c}$ for some $c$. Q.E.D.

\section{Classification of SOlutions}

In this section we shall prove Theorems $1.5,1.6$ and 1.7.

Proof of Theorem 1.5. Let $u$ be a solution of (1.1). Suppose that $u \neq U$ on $\mathbb{R}^{n}$. Then from Theorem 2.3,u<U on $\mathbb{R}^{n}$. Let $w=U-u$. Then $w$ satisfies

$$
\Delta w-d(x) w=0,
$$


where

$$
\begin{aligned}
d(x) & =-K(x) \frac{e^{2 U(x)}-e^{2 u(x)}}{U(x)-u(x)} \\
& \leq-K(x) \cdot 2 e^{2 U(x)} \leq C|x|^{-2}
\end{aligned}
$$

for $|x|$ large. Let $|y|=R$ and $R$ be large. Consider (5.1) in the ball $B_{R / 2}(y)=$ $\left\{x \in \mathbb{R}^{n}|| x-y \mid<R / 2\right\}$. Using the Harnack inequality [GT, p. 199], we have

$$
\sup _{x \in B_{R / 8}(y)} w(x) \leq C \inf _{x \in B_{R / 8}(y)} w(x)
$$

where $C$ is a constant independent of $R$. Thus there exists a constant $C>1$ such that

$$
\sup _{|x|=r} w(x) \leq C \inf _{|x|=r} w(x)
$$

for $r$ large.

Now suppose that $w>0$ is bounded. We have

$$
\Delta w(x)=-K(x)\left[e^{2 U(x)}-e^{2 u(x)}\right]=-K(x) \frac{\left[e^{2 U(x)}-e^{2 u(x)}\right]}{w^{2}} w^{2} .
$$

Let $M$ be a bound of $w$. Then

$$
\begin{aligned}
-K(x) \frac{e^{2 U}-e^{2 u}}{w^{2}} & =-K(x) e^{2 u}\left[\frac{e^{2 w}-1}{w^{2}}\right] \\
& \geq-K(x) e^{2(U(x)-M)}\left[\frac{e^{2 w}-1}{w^{2}}\right] \\
& \geq C[-K(x)] e^{2 U(x)}
\end{aligned}
$$

for some constant $C>0$. Thus

$$
\Delta w \geq C[-K(x)] e^{2 U(x)} w^{2} \quad \text { on } \mathbb{R}^{n} .
$$

Since $-K(x) e^{2 U(x)} \sim|x|^{-2}(\log |x|)^{-1}$ at $\infty$, we conclude from [CL] that (5.6) does not possess any solution on $\mathbb{R}^{n}$. Hence $w$ is unbounded. Let $\left\{x_{m}\right\}$ be a sequence such that $\left|x_{m}\right| \rightarrow \infty$ as $m \rightarrow \infty$ and $w(x) \rightarrow \infty$ as $m \rightarrow \infty$. From (5.4), we conclude that inf $|x|=\left|x_{m}\right| w(x) \rightarrow \infty$. Let $\bar{w}(r)$ be the spherical mean of $w$ on $|x|=r$. Then since $w$ is a subharmonic function on $\mathbb{R}^{n}, \bar{w}$ is a convex function of $r^{2-n}$. Hence we conclude that $\bar{w}(r) \rightarrow \infty$ as $r \rightarrow \infty$. Again from (5.4), we conclude that $w(x) \rightarrow \infty$ as $|x| \rightarrow \infty$.

Now $w$ satisfies $\Delta w=-K\left[e^{2 U}-e^{2 u}\right]$. Hence $\bar{w}(r)$ satisfies

$$
\bar{w}^{\prime \prime}+\frac{n-1}{r} \bar{w}^{\prime}=-\overline{K\left[e^{2 U}-e^{2 u}\right]}=-\overline{K e^{2 U}\left[1-e^{-2 w}\right]} \geq C \overline{\left[-K e^{2 U}\right]},
$$

where "-" means the spherical average on $|x|=r$.

From the assumptions of $K$ and the asymptotic behaviors of $U$, we have $-\overline{K e^{2 U}} \geq C r^{-2}$ or $-\overline{K e^{2 U}} \geq C r^{-2}(\log r)^{-1}$ for $r$ large. Hence we conclude that $\bar{w}(r) \geq C \log r$ or $\bar{w}(r) \geq C \log \log r$ for $r$ large. By (5.4) again, we have $w(x) \geq C \log |x|$ or $w(x) \geq C \log \log |x|$ for $|x|$ large. Thus $u(x) \leq$ $U(x)-C \log |x|$ or $u|x| \leq U|x|-C \log \log |x|$ for $|x|$ large. Thus

$$
\int_{\mathbb{R}^{n}} \frac{-K(y)}{|y|^{n-2}} e^{2 u(y)} d y<\infty .
$$


From [HK], $u$ is bounded and hence by Theorem $1.1(\delta), u=u_{c}$ for some $c \in \mathbb{R}$. Q.E.D.

Proof of Theorem 1.6. Let $u$ be a solution of (1.1) with a lower bound $(-M)$. Let $v=u+M+1$. Then $v \geq 1$ and satisfies

$$
\begin{aligned}
\Delta v & =-K(x) e^{2 u}=[-K(x)] e^{-2(M+1)} e^{2 v} \\
& =[-K(x)] e^{-2(M+1)} \frac{e^{2 v}}{v} v=d(x) v,
\end{aligned}
$$

where $d(x)=[-K(x)] e^{-2(M+1)} e^{2 v} / v$. For $|x|$ large, if $1 \leq v(x) \leq \frac{1}{2} U(x)$, then

$$
d(x) \leq[-K(x)] e^{-2(M+1)} \frac{e^{U(x)}}{1} \leq \frac{C}{|x|^{2}} .
$$

If $v(x) \geq \frac{1}{2} U(x)$, then

$$
d(x) \leq[-K(x)] e^{-2(M+1)} \frac{e^{2(U(x)+M+1)}}{\frac{1}{2} U(x)} \leq \frac{C}{|x|^{2}} .
$$

Thus as in the proof of (5.4), we conclude that

$$
\sup _{|x|=r} v(x) \leq C \inf _{|x|=r} v(x)
$$

for $r$ large for some constant $C \geq 1$.

Now suppose that $u$ is bounded also from above. Then from Theorem $1.1(\beta),(\delta), u=u_{c}$ for some $c \in \mathbb{R}$. If $u$ is not bounded from above, then there exists a sequence $\left\{x_{m}\right\}$ such that $\left|x_{m}\right| \rightarrow \infty$ as $m \rightarrow \infty$ and $u\left(x_{m}\right) \rightarrow \infty$ as $m \rightarrow \infty$. Hence $v\left(x_{m}\right) \rightarrow \infty$. From (5.8) and the same arguments as in the proof of Theorem 1.5, we conclude that $\lim _{|x| \rightarrow \infty} v(x)=\infty$. Hence $u(x) \rightarrow \infty$ as $|x| \rightarrow \infty$.

Let $K_{2}=K_{2}(r)$ be a function such that $K_{2}(|x|) \leq K(x)$ for all $x \in \mathbb{R}^{n}$ and $\left|K_{2}(|x|)\right| \sim|K(x)|$ for $|x|$ large. Let $v_{c}(r)$ and $V(r)$ be the solution of

$$
v^{\prime \prime}+\frac{n-1}{r} v^{\prime}+K_{2}(r) e^{2 v}=0
$$

satisfying $v_{c}(r) \rightarrow c$ as $r \rightarrow \infty$ and $v_{c}(r) \rightarrow V(r)$ as $c \rightarrow \infty$. From Theorem 1.4 , these are the radial solutions of $\Delta v+K_{2} e^{2 v}=0$ on $\mathbb{R}^{n}$.

Now by the maximum principle, it is easy to see that $v_{c}(|x|) \leq u(x), x \in \mathbb{R}^{n}$. Hence $V(|x|) \leq u(x), x \in \mathbb{R}^{n}$. This proves that $u(x) \geq U(x)-C, x \in \mathbb{R}^{n}$, for some $C$. As in the proof of Theorem 1.5, we conclude that $u=U$. Q.E.D.

Finally, we give the proof of Theorem 1.7.

Proof of Theorem 1.7. Let $h$ be a harmonic function on $\mathbb{R}^{n}$. Consider the problem

$$
\Delta w+\left(K e^{2 h}\right) e^{2 w}=0 \quad \text { on } \mathbb{R}^{n} .
$$

From Theorem 1.1 $(\beta),(5.10)$ possesses a unique solution $w_{c}$ with $w_{c}(x) \rightarrow c$ as $|x| \rightarrow \infty$ for every $c$. Hence $u=h+w_{c}$ is the unique solution satisfying (1.18). This proves (i). 
Now let $u$ be a solution of (1.1). Then since $K \leq 0$ and $K$ has compact support, $u$ is a subharmonic function such that

$$
\int_{\mathbb{R}^{n}} \frac{|+\Delta u(y)|}{|y|^{n-2}} d y=\int_{\mathbb{R}^{n}} \frac{|K(y)|}{|y|^{n-2}} e^{2 u(y)} d y<\infty .
$$

From [HK], we conclude that there exist a harmonic function $h$ and a constant $c$ such that (1.18) holds. This proves (ii). Q.E.D.

\section{REFERENCES}

[CL] K.-S. Cheng and J.-T. Lin, On the elliptic equations $\Delta u=K u^{\sigma}$ and $\Delta u=K e^{2 u}$, Trans. Amer. Math. Soc. 304 (1987), 639-668.

[CN1] K.-S. Cheng and W.-M. Ni, On the structure of the conformal Gaussian curvature equation on $\mathbb{R}^{2}$, Duke Math. J. 62 (1991), 721-737.

[CN2] - On the structure of the conformal scalar curvature equation on $\mathbb{R}^{n}$, Indiana Univ. Math. J. (to appear).

[GT] D. Gilbarg and N. S. Trudinger, Elliptic partial differential equations of second order, 2nd ed., Springer-Verlag, 1983.

[HK] W. K. Hayman and P. B. Kennedy, Subharmonic functions, vol. 1, London Math. Soc. Monographs, no. 9, Academic Press, London, 1976.

[K] J. Kazdan, Prescribing the curvature of a Riemannian manifold, CBMS Regional Conf. Ser. in Math., no. 57, Amer. Math. Soc., Providence, R.I., 1985.

[KO] T. Kusano and S. Oharu, Bounded entire solutions of second order semilinear elliptic equation with application to a parabolic initial value problem, Indiana Univ. Math. J. 34 (1985), 8589.

[LC] J.-T. Lin and K.-S. Cheng, Examples of solution for semilinear elliptic equations, Chinese J. Math. 15 (1987), 43-59.

[N1] W.-M. Ni, On the elliptic equation $\Delta u+K e^{2 u}=0$ and conformal metrics with prescribed Gaussian curvatures, Invent. Math. 66 (1982), 343-352.

[N2] - On the elliptic equation $\Delta u+K u^{(n+2) /(n-2)}=0$, its generalizations and applications in geometry, Indiana Univ. Math. J. 31 (1982), 493-529.

[O] O. A. Oleinik, On the equation $\Delta u+K(x) e^{u}=0$, Russian Math. Surveys 33 (1978), 243-244.

Institute of Applied Mathematics, National Chung Cheng University, Chiayi, Taiwan 62117, Republic OF China

Institute of Applied Mathematics, National Tsing Hua University, Hsinchu, Taiwan 30043, RePUBLIC OF ChiNA 